\title{
Refactoring Sequential Java Code for Concurrency via Concurrent Libraries
}

\author{
Danny Dig, John Marrero, Michael D. Ernst \\ Massachusetts Institute of Technology \\ Computer Science and Artificial Intelligence Laboratory \\ \{dannydig,marrero,mernst\}@ csail.mit.edu
}

\begin{abstract}
Parallelizing existing sequential programs to run efficiently on multicores is hard. The Java 5 package java.util.concurrent (j.u.c.) supports writing concurrent programs: much of the complexity of writing threads-safe and scalable programs is hidden in the library. To use this package, programmers still need to reengineer existing code. This is tedious because it requires changing many lines of code, is error-prone because programmers can use the wrong APIs, and is omission-prone because programmers can miss opportunities to use the enhanced APIs.

This paper presents our tool, CONCURRENCER, which enables programmers to refactor sequential code into parallel code that uses j.u.c. concurrent utilities. ConcurRENCER does not require any program annotations, although the transformations are very involved: they span multiple program statements and use custom program analysis. A find-and-replace tool can not perform such transformations. Empirical evaluation shows that CONCURRENCER refactors code effectively: CONCURRENCER correctly identifies and applies transformations that some open-source developers overlooked, and the converted code exhibits good speedup.
\end{abstract}

\section{Introduction}

Users expect that each new generation of computers runs their programs faster than the previous generation. The computing hardware industry's shift to multicore processors demands that programmers find and exploit parallelism in their programs, if they want to reap the same performance benefits as in the past.

In the multicore era, a major programming task is to retrofit parallelism into existing sequential programs. It is arguably easier to design a program with concurrency in mind than to retrofit concurrency later $[8,12]$. However, most desktop programs were not designed to be concurrent, so programmers have to refactor existing sequential programs for concurrency. It is easier to retrofit concurrency than to rewrite, and this is often possible.

The dominant paradigm for concurrency in desktop programs is multithreaded programs where shared-memory accesses are protected with locks. However, programming with locks is very error-prone: too many locks can greatly slow down or even deadlock an application, while too few locks result in data races.

Java 5's java.util.concurrent (j.u.c.) package supports writing concurrent programs. Its Atomic* classes offer thread-safe, lock-free programming over single variables. Its thread-safe abstract data types (e.g., ConcurrentHashMap) are optimized for scalability.

Java 7 will contain a framework Fork/Join Task (FJTask) [7, 10] for fine-grained parallelism. Many computationally-intensive problems take the form of recursive divide-and-conquer. Classic examples include sorting (mergesort, quicksort), searching, and many data structure or image processing algorithms. Divide-and-conquer algorithms are good candidates for parallelization since the subproblems can be solved in parallel.

However, in order to benefit from Java's concurrent utilities and frameworks, the Java programmer needs to refactor existing code. This is tedious because it requires changing many lines of code. For example, the developers of six widely used open-source projects changed 1019 lines when converting to use the j.u.c utilities. Second, manual refactoring is error-prone because the programmer can choose the wrong APIs among slightly similar APIs. In the above-mentioned projects, the programmers four times mistakenly used getAndIncrement API methods instead of incrementAndGet, which can result in off-by-one values. Third, manual refactoring is omission-prone because the programmer can miss opportunities to use the new, more efficient API methods. In the same projects, programmers missed 41 such opportunities.

This paper presents our approach for incrementally retrofitting parallelism through a series of behaviorpreserving program transformations, namely refactorings. Our tool, CONCURRENCER, enables Java programmers to quickly and safely refactor their sequential programs to use 
j.u.c. utilities. Currently, Concurrencer supports three refactorings: (i) CONVERT Int to Atomicinteger, (ii) Convert hashMap to ConcurrentHashMap, and (iii) ConVERt ReCursion to FJTASк. We previously cataloged [4] the transformations that open-source developers used to parallelize five projects. We found that the first two refactorings were among the most commonly used in practice.

The first refactoring, Convert Int to Atomicinteger, enables a programmer to convert an int field to an AtomicInteger, a utility class that encapsulates an int value. The encapsulated field can be safely accessed from multiple threads, without requiring any synchronization code. Our refactoring replaces all field accesses with calls to AtomicInteger's thread-safe APIs. For example, it replaces expression $f=f+3$ with $f$.addAndGet (3) which executes atomically.

The second refactoring, Convert HashMaP to CONCURRENTHASHMAP, enables a programmer to convert a HashMap field to ConcurrentHashMap, a thread-safe, highly scalable implementation for hash maps. Our refactoring replaces map updates with calls to the APIs provided by ConcurrentHashMap. For example, a common update operation is (i) check whether a map contains a certain $k e y$, (ii) if not present, create the value object, and (iii) place the value in the map. Concurrencer replaces such an updating pattern with a call to ConcurrentHashMap's put IfAbsent which atomically executes the update, without locking the entire map. The alternative is for programmer to place a lock around the updating code, but this is error-prone and the map's lock degrades the map's performance under heavy lock-contention.

The third refactoring, CONvert Recursion to FJTAsk, enables a programmer to convert a sequential divide-and-conquer algorithm to a parallel algorithm. The parallel algorithm solves the subproblems in parallel using the FJTask framework. Using the skeleton of the sequential algorithm, CoNCURRENCER extracts the sequential computation into tasks which run in parallel and dispatches these tasks to the FJTask framework.

Typically a user would first make a program thread-safe, i.e., the program has the same semantics as the sequential program even when executed under multiple threads, and then make the program run concurrently under multiple threads. CONCURREncer supports both kinds of refactorings: the first two refactorings are "enabling transformations": they make a program thread-safe. The third refactoring makes a sequential program run concurrently.

The transformations performed by these refactorings are involved: they require matching certain code patterns which can span several non-adjacent program statements, and they require program analysis which uses data-flow information. Such transformations can not be safely executed by a findand-replace tool.
This paper makes the following contributions:

- Approach. We present an approach for retrofitting parallelism into sequential applications through automated, but human-initiated, program transformations. Since the programmer is expert in the problem domain, she is the one most qualified to choose the code and the program transformation for parallelizing the code.

- Tool. We implemented three transformations for using thread-safe, highly scalable concurrent utilities and frameworks. Our tool, Concurrencer, is conveniently integrated within Eclipse's refactoring engine. ConcurRENCER can be downloaded from: http://refactoring.info/tools/Concurrencer

- Empirical Results. We used Concurrencer to refactor the same code that the open-source developers of 6 popular projects converted to AtomicInteger and ConcurrentHashMap. By comparing the manually vs. automatically refactored output, we found that ConCURRENCER applied all the transformations that the developers applied. Even more, CONCURRENCER avoided the errors which the open-source developer committed, and CONCURRENCER identified and applied some transformations that the open-source developers omitted. We also used CONCURRENCER to parallelize several divideand-conquer algorithms. The parallelized algorithms perform well and exhibit good speedup. These experiences show that CONCURRENCER is useful.

\section{Convert Int to AtomicInteger}

\subsection{AtomicInteger in Java}

The Java 5 class library offers a package j.u.c. atomic that supports lock-free programming on single variables.

The package contains wrapper classes over primitive variables, for example, an AtomicInteger wraps an int value. The main advantage is that update operations execute atomically, without blocking. Internally, AtomicInteger employs efficient machine-level atomic instructions like Compare-and-Swap that are available on contemporary processors. Using AtomicInteger, the programmer gets both thread-safety (built-in the Atomic classes) and scalability (the lock-free updates eliminate lock-contention under heavy accesses [8]).

\subsection{Code Transformations}

A programmer who wanted to use Concurrencer to make all accesses to an int thread-safe would start by selecting the field and invoking the CONVERT INT TO ATOMICINTEGER refactoring. CONCURRENCER changes the declaration type of the int 


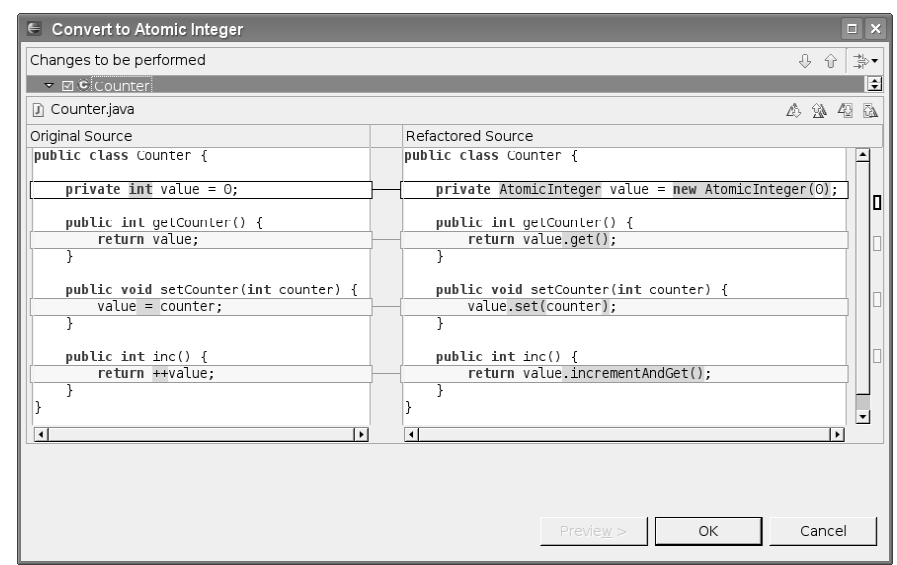

Figure 1. Using CONCURRENCER to convert an int to AtomicInteger in Apache Tomcat. Left/right shows code before/after refactoring.

field to AtomicInteger and replaces all field updates with their equivalent atomic API methods in AtomicInteger.

Figure 1 shows how ConcurRencer refactors some code from Apache Tomcat. We use this example to illustrate various code changes.

Initialization. Because the refactored value field is an AtomicInteger object, CONCURRENCER initializes it in the field initializer (otherwise a NullPointerException is thrown the first time that a method is invoked on value). CONCURRENCER uses the field initializer expression or the implicit expression ' 0 '.

Read/Write Accesses. Concurrencer replaces read access (e.g., in method getCounter()) with a call to AtomicInteger's get (). This has the same type int as the expression being replaced, so clients are unaffected. Since the mutable Atomic Integer does not escape its containing class Counter, a client can mutate the field only through the Counter's methods.

ConcurRencer replaces write accesses (e.g., in method setCounter) with a call to AtomicInteger's set () .

Update Expressions. There are three kinds of update expressions: infix (e.g., $f=f+2$ ), prefix (e.g., $++f)$, and postfix (e.g., $f++$ ). CONCURRENCER rewrites an infix update expression using a call to the atomic addAndGet (int delta).

Concurrencer rewrites a prefix update expression with a call to the atomic incrementAndGet () (e.g., method inc () in Fig. 1). It rewrites a postfix expression with a call to the atomic getAndIncrement (). CONCURRENCER similarly rewrites the decrement expressions.

AtomicInteger only provides APIs for replacing infix expressions involving the + operator. CONCURRENCER converts an infix expressions that use the - operator to an addition expression (e.g., it converts $f=f-5$ to f.addAndGet (-5)). If AtomicInteger had methods
divideAndGet or multiplyAndGet, then ConCuRRENCER could use them. In such cases, CONCURRENCER warns the user that the update expression cannot be made thread-safe, other than by using locks.

Synchronization. ConCURRENCER converts both a sequential program into one which is thread-safe, and also an already-concurrent program into one which is *more* concurrent. If the original code contains synchronized accesses to the int field, Concurrencer tries to remove the synchronization since this becomes superfluous after the conversion to Atomic Integer (thread-safety is built-in the AtomicInteger). CONCURRENCER only removes the synchronization if the code after refactoring contains exactly one call to the atomic APIs. Otherwise, a context switch can still occur between two consecutive calls to atomic APIs, For example, ConCURRENCER removes the synchronization in the code fragment below:

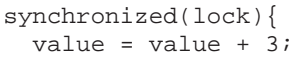

but does not remove synchronization for the code fragment below:

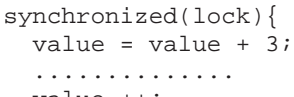

Similarly, ConcurRencer only removes the synchronization if the synchronized block contains updates to one single field. Since the AtomicInteger ensures thread-safety for only one single field, it is of no help in cases when the program needs to maintain an invariant over multiple fields. For example a ValueRange object needs to ensure that its max int field is always greater than its min int field. To handle this, Concurrencer would need multivariable thread-safe container classes, currently not provided by j.u.c.

\section{Convert HashMap to Concurren- tHashMap}

\subsection{ConcurrentHashMap in Java}

The j.u.c. package contains several concurrent collection classes. ConcurrentHashMap is a thread-safe implementation of HashMap.

Before the introduction of j.u.c., a programmer could create a thread-safe HashMap using a synchronized wrapper over a HashMap (e.g., Collections.synchronizedMap (aMap)). The synchronized HashMap achieves its thread-safety by protecting all accesses to the map with a common lock. This results in poor concurrency when multiple threads contend for the lock. 
ConcurrentHashMap uses a more scalable locking strategy. All readers run concurrently, and lock-striping allows a limited number of writers to update the map concurrently. The implementation uses $N$ locks (the default value is 16), each of them guarding a part of the hash buckets. Assuming that the hash function spreads the values well, and that keys are accessed randomly, this reduces the contention for any given lock by a factor of $\mathrm{N}$.

ConcurrentHashMap implements the Map interface, therefore it includes the API methods offered by Hashmap. In addition, it contains three new APIs putIfAbsent(key, value), replace(key, oldValue, newValue), and a conditional remove (key, value). Each of these new APIs:

- supersedes several calls to Map operations, and

- executes atomically.

For example putIfAbsent (i) checks whether the map contains a given key, and (ii) if absent, inserts the $\langle k e y$, value $\rangle$ entry.

Replacing a synchronized HashMap with ConcurrentHashMap offers dramatic scalability improvements [8].

\subsection{Code Transformations}

A programmer who wanted to use ConcurRencer to make all accesses to an HashMap thread-safe would start by selecting the field and invoking the CONVERT HASHMAP TO CONCURRENTHASHMAP refactoring.

Initialization and Accesses. CONCURRENCER changes the declaration and the initialization of the field. Because HashMap and ConcurrentHashMap implement the same interface (Map), initialization and map accesses remain largely the same.

Map Updates. Concurrencer detects update code patterns and replaces them with the appropriate Concurrent HashMap API method.

The patterns have a similar structure: (i) check whether the map contains a certain key, and (ii) depending on the result, invoke one of put (key, value) or remove (key). This structure can have small variations. For instance, the check can invoke containskey, get, or an equality check using get. A temporary variable might hold the result of the check (like in Fig. 2). Concurrencer handles all combinations among these map update variations. Although these are the most common variations we have seen in real code, there might be other variations. Failing to convert those updates does not break user code; it only misses the opportunity to eliminate synchronization.

Fig. 2 illustrates one of the simplest transformations for using putifabsent. In order to identify the potential usage of put IfAbsent, CONCURRENCER searches for conditional code which checks whether a certain key is not present in the cache Map field. If a put method call in the same conditional body uses the same key, ConCURRENCER has identified a potential usage of put IfAbsent. Next, CONCURRENCER replaces the two calls to the older APIs (get and put) with one call to put IfAbsent which executes atomically, without locking the entire map. The alternative is to protect the pair get/put with one global lock, but this alternative greatly reduces the application's scalability since the lock would prevent any other concurrent access to the map.

In the example in Fig. 2, the value to be placed in the $\langle k e y, v a l u e\rangle$ map entry is simply created by invoking a constructor. However, in many cases the creational code for the newly inserted value is much more involved. Since the value to be inserted must be available before invoking putIfAbsent, in Fig. 3, ConcurRENCER extracts the creational code into a creational method (createTimezoneList) and calls it to compute the value.

CONCURREnCER performs a data-flow analysis to find out if the created value is read after the call to put. If so, the created value is stored so that it can be accessed later. The example in Fig. 3 shows another trait: the timeZoneList value is also written in the conditional code. The original getTimezoneList method returns either the null value, or the new value created in the conditional code. To preserve these semantics, CONCURRENCER conditionally assigns the newly created value to the timezoneLists variable. The new conditional expression checks whether the call to putIfAbsent was successful: if the call succeeded, putIfAbsent returns null, otherwise it returns the previous value associated with the key. The refactored code uses the return status to decide whether to store the newly created value into the timezoneList variable.

Before calling the putIfAbsent method, the value to be inserted must be available. Therefore, in the refactored code, the creational code is executed regardless of whether the new value is placed into the map. The refactored method has different semantics if the creational code has side effects (e.g., logging). Concurrencer checks whether the creational method has side effects, and if so, it warns the user.

We implemented a conservative analysis for determining side-effects. CONCURRENCER warns the user when the creational method assigns to fields or method arguments and when a method is called on a field or local variable. A constructor call is a special case of method call because fields nay be assigned inside the constructor.

An alternative to calling the creational method before putIfAbsent is to extract the creational code into a Future object, a j.u.c. utility class which represents the result of a future computation. In this case, the result of creational code will be retrieved only the first time when the programmer calls get on the Future object. ConCURRENCER could change all map.get (value) accesses to 


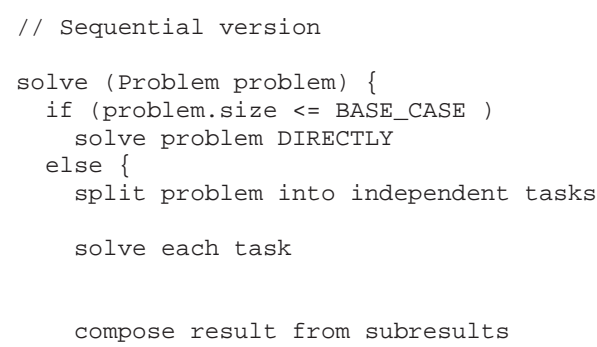

Figure 4. Pseudocode for divide-and-conquer algorithm. Left hand side shows the sequential version, right hand side shows the parallel version.

eral divide-and-conquer algorithm. In the parallel version, if the problem size is smaller than a threshold, the problem is solved using the sequential algorithm. Otherwise, the problem is split into independent parts, these are solved in parallel, then the algorithm waits for all computations to finish and composes the result from the subresults.

Given the nature of divide-and-conquer algorithms, tasks that run in parallel should have the following characteristics:

- they are CPU-bound not I/O-bound, thus they do not block on $\mathrm{I} / \mathrm{O}$

- depending on the sequential threshold, many tasks (e.g. tens of thousands) can be spawned by the recursion branches

- they only need to synchronize when waiting for subtasks to complete

Given these properties, threads are not a good vehicle for running such tasks. Threads have high overhead (creating, scheduling, destroying) which might outperform the useful computation. Therefore Java 7 introduces ForkJoinTask, a lighter-weight thread-like entity. A large number of such tasks may be hosted by a pool containing a small number of actual threads. The task scheduling is based on workstealing $[6,13]$ : idle worker threads "steal" work from busy threads. The framework avoids contention for the data structures that hold the scheduling and ensures that each theft acquires a large chunk of work, thus making stealing infrequent. It is this effective scheduling that keeps all the cores busy with useful computation.

The most important API methods in ForkJoinTask are: fork (Task) which spawns the execution of a new task in parallel, join (Task) which blocks the current computation until the task passed as an argument finished, forkJoin(Tasks) which is syntactic sugar for calling fork and then join, and compute which is the hook-up method invoked by the framework when executing each task. compute implements the main computation performed by the task.

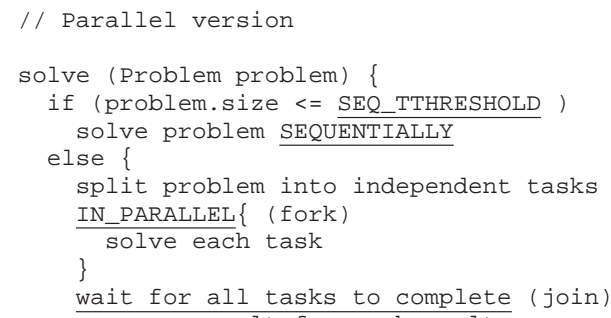
ForkJoinTask has several subclasses for different patterns of computation. RecursiveAction is the proper choice for the recursive tasks used in divideand-conquer computations. The framework also defines ForkJoinExecutor, an object which executes ForkJoinTask computations using a pool of worker threads.

\subsection{Code Transformations}

CONCURRENCER converts a recursive divide-and-conquer algorithm to one which runs in parallel using the FJTask framework. The programmer need only select the divide-and-conquer method and supply the SEQUENTIAL THRESHOLD parameter that determines when to run the sequential version of the algorithm. Using this user-supplied information, CONCURRENCER automatically performs all transformations.

We made a design choice to keep the original interface of the recursive method unchanged, so that an outside client would still invoke the method as before. The fact that the refactored method uses the FJTask framework is an implementation detail, hidden from the outside client.

We illustrate the transformations that CONCURRENCER performs on a classic merge sort algorithm. The left-hand side of Fig. 5 shows the original, sequential version of the merge sort algorithm. The sort method takes as input the array to be sorted and returns the sorted array. The algorithm starts with the base case. In the recursion case, it copies the first half of the array and the second half of the array, sorts both halves, and merges them (code for merge not shown).

Creating the ForkJoinTask. Concurrencer creates a RecursiveAction class, which is a subclass of ForkJoinTask. This class encapsulates the parallel computation of the original recursive method, thus CONCURRENCER names this class by adding the "Impl" suffix to the name of the original recursive method.

Since the compute hook-up method neither takes any arguments, nor returns a value, the SortImpl has fields for the input arguments and the result of the computation. For each formal parameter of the original recursive method, 
27). If the original method returned a value, ConCURRENCER saves this value in the result field.

Second, CONCURRENCER replaces the recursive calls with creation of new RecursiveAction objects (lines 35, 36). The arguments of the recursive call are passed as arguments to the constructor of the RecursiveAction. CONCURRENCER stores the newly created tasks into local variables, named task1, task2, etc.

Third, CONCURREncer executes the parallel tasks and then assembles the result from the subresults of the tasks. ConCURRENCER invokes the forkJoin method while passing the previously created tasks as arguments. CONCURRENCER places the forkJoin method after the last creation of RecursiveAction (line 37). Then Concurrencer saves the subresults of the parallel tasks into local variables. If the original recursive method used local variables to store the results of the recursive calls, CONCURRENCER reuses the same variables (lines 38,39 ). Subsequent code can thus use the subresults to assemble the final result (line 40). Lastly, ConCURRENCER assigns to the result field the combined subresults (line 41).

Reimplementing the recursive method. CONCURRENCER changes the implementation of the original recursive method to invoke the F JTask framework (lines 10-13). CoNCURRENCER creates an executor object and initializes it with the number of threads to be used by the worker thread pool. The number of threads is equal with the number of available processors (found at runtime). The Java runtime system ensures that each processor will be assigned one worker thread. Since the divide-and-conquer algorithm is CPUbound, creating more threads will not speed up the execution; on the contrary, it might slow the execution due to thread scheduling overhead. CONCURRENCER creates a new task and initializes it with the array to be sorted, then it passes the task to the executor. invoke blocks until the computation finishes. Once the computation finished, the sorted array available in the result field is returned (line 14).

Discussion. CONCURREncer handles several variations on how the subresults are combined to form the end result. For example, the subresults of the recursive calls might not be stored in temporary variables, but they might be combined directly in expressions. For example, a fibonacci function returns:

return fibonacci $(n-1)+$ fibonacci $(n-2)$.

CONCURRENCER creates and executes the parallel tasks as before, and during the subresult combination phase it uses the same expression to combine the subresults:

result $=$ task1.result + task2.result

With respect to where the recursive method stores the result, there can be two kinds of recursive methods: (i) recursive methods which return a value, the result, and (ii) recursive methods which do not return any value, but they mutate at least one of the arguments to hold the result of the computation.

Fig. 5 is an example of the first kind of computation. The transformations for recursive methods which mutate one of their arguments to store the result are similar with the ones presented above, even slightly simpler, i.e., ConCURRENCER does not generate the code involving the result field.

\section{Evaluation}

Research Questions. To evaluate the effectiveness of CONCURREnCER, we answered the following questions:

- Q1: Is Concurrencer useful? More precisely, does it ease the burden of making sequential code thread-safe and of running concurrent tasks in parallel?

- Q2: With respect to thread-safety, how does the manually refactored code compare with code refactored with CONCURRENCER in terms of using the correct APIs and identifying all opportunities to replace field accesses with thread-safe API calls?

- Q3: With respect to running concurrent tasks in parallel, is the refactored more efficient than the original sequential code?

We evaluated ConcurRencer's refactorings in two ways. For code that had already been refactored to use Java 5's AtomicInteger and Concurrent HashMap we compared the manual refactoring with what CONCURRENCER would have done. This answers the first two questions. For ConVERT RECURSION TO FJTASK, since FJTask is scheduled for Java 7's release, we could not find existing codebases using FJTask. We used CONCURRENCER to refactor several divideand-conquer algorithms, and we answer first and third question.

\subsection{Methodology}

Setup for Convert int to Atomicinteger and Convert HASHMAP TO CONCURRENTHASHMAP.

Table 1 lists 6 popular, mature open-source projects that use AtomicInteger and Concurrent HashMap. We used the head versions available in their version control system as of June 1, 2008.

We used CONCURRENCER to refactor the same fields that open-source developers refactored to AtomicInteger or ConcurrentHashMap. We compare the code refactored with CONCURRENCER against code refactored by hand. We look at places where the two refactoring outputs differ, and quantify the number of errors (i.e., one of the outputs uses the wrong concurrent APIs) and the number of omissions (i.e., 


\begin{tabular}{l|l|c|c|c} 
refactoring & in project & $\begin{array}{c}\text { \# of } \\
\text { refactorings }\end{array}$ & $\begin{array}{c}\text { LOC } \\
\text { changed }\end{array}$ & $\begin{array}{c}\text { LOC CONCURRENCER } \\
\text { can handle }\end{array}$ \\
\hline Convert Int & MINA & 5 & 21 & 21 \\
To AtomicInteger & Tomcat & 5 & 26 & 26 \\
& Struts & 0 & 0 & 0 \\
& GlassFish & 15 & 60 & 60 \\
& JaxLib & 29 & 240 & 240 \\
\hline Convert HashMap & Zimbra & 10 & 54 & 54 \\
To ConcurrentHashMap & MINA & 6 & 14 & 14 \\
& Tomcat & 0 & 0 & 0 \\
& Struts & 6 & 68 & 86 \\
& GlassFish & 14 & 86 & 62 \\
\hline Total for AtomicInteger & JaxLib & 7 & 62 & 377 \\
and ConcurrentHashMap & Zimbra & 44 & 388 & $\mathbf{9 6 8}$ \\
\hline Convert Recursion & mergeSort([15]) & $\mathbf{1 4 1}$ & $\mathbf{1 0 1 9}$ & \\
to FJTask & fibonacci( [13]) & 1 & 36 & 36 \\
& maxSumConsecutive( [13]) & 1 & 68 & 25 \\
\hline Total for FJTask & matrixMultiply ([5,13,15]) & 1 & 108 & 68 \\
& quickSort(Zimbra) & 1 & 35 & 30 \\
\hline & maxTreeDepth(Eclipse) & 1 & 30 & $\mathbf{3 0 2}$
\end{tabular}

Table 1. Programs used as case studies for Convert Int to ATOMICINTEGER, CONVERT HASHMAP to CONCURRENTHASHMAP, and CONVERT RECURSION to FJTASK refactorings. Last two columns show LOC changed due to refactoring, and how many LOC can be changed by CONCURRENCER.

the refactored output could have used a concurrent API, but it instead uses the obsolete, lock-protected APIs).

For AtomicInteger we were able to find both the version with the int field and the version with AtomicInteger field, thus we use the version with int as the input for Concurrencer. For Convert hashmap to ConCURRENTHASHMAP we were not able to find the versions which contained HashMap. It seems that those projects were using Concurrent HashMap from the first version of the file. In those cases we manually replaced only the type declaration of the Concurrent HashMap field with HashMap; then we ran CONCURRENCER to replace HashMap updates with the thread-safe APIs (putIfAbsent, replace, and delete) in ConcurrentHashMap.

Setup for Convert Recursion to FJTASK.

We used Concurrencer to parallelize several divide-andconquer algorithms. We use two sets of inputs: (i) classic divide-and-conquer algorithms used in others' evaluations [5, 13, 15], and (ii) divide-and-conquer algorithms from real projects.

Table 1 shows the input programs. maxSumConsecutive takes an array of positive and negative numbers and computes the subsequence of consecutive numbers whose sum is maximum. matrixMultiply multiplies two matrices. maxTreeDepth computes the depth of a binary tree.

The interested reader can find the input and the refactored programs on CONCURRENCER's webpage.

\subsection{Q1: Is CONCURRENCER useful?}

The top part of Table 1 show the number of refactorings that open-source developers performed in the selected real world projects. The penultimate column shows how many lines of code were manually changed during refactoring. Using ConCURRENCER, the developers would have saved editing 968 lines of code; instead they would have had to only change 51 lines not currently handled by CONCURRENCER.

The bottom part of Table 1 show the LOC changed when converting the original recursive algorithm to one which uses the FJTask framework. To do the manual conversion, it took the first author an average of 30 minutes for each conversion. This includes also the debug time to make the parallelized algorithm work correctly. Using CONCURRENCER, the conversion was both correct and took less than $10 \mathrm{sec}-$ onds. Doing the conversion with Concurrencer saves the programmer from changing 302 LOC.

\subsection{Q2: How does manually and automat- ically refactored code compare?}

CONCURRENCER applied all the correct transformations that the open-source developers applied. We noticed several cases where CONCURRENCER outperforms the developers: CONCURRENCER produces the correct code, or it identifies more opportunities for using the new, scalable APIs.

For Convert Int to Atomicinteger, we noticed cases where 
the developers used the wrong APIs when they refactored by hand. We noticed that developers erroneously replaced infix expressions like $++f$ with $f$.getAndIncrement (), which is the equivalent API for the postfix expression f++. They should have replaced $++f$ with f.incrementAndGet (). Table 2 shows that the opensource developers made 4 such errors, where Concurrencer made no error. The erroneous usage of the API can cause an "off-by-one" value if the result is read in the same statement which performs the update. In the case studies, the incremented value is not read in the same statement which performs the update.

For Convert hashMap to Concurrenthashmap we noticed cases when the open-source developers or ConCURRENCER omitted to use the new atomic putIfAbsent and conditional delete operations, and instead use the old patterns involving synchronized, lock-protected access to put and delete. Although the refactored code is thread-safe, it is non-optimal for these lines of code because it locks the whole map for the duration of update operations. In contrast, ConcurrentHashMap's new APIs offers better scalability because they do not lock the whole map.

Table 3 shows the number of such omissions in the case-study projects. We manually analyzed all the usages of put or delete and compiled a list of all the places where those usages could have been replaced with the new put IfAbsent, replace, or conditional delete. We found that the open-source developers missed many opportunities to use the new APIs. This intrigued us, since the studied projects are all developed professionally, and are known to be of high-quality (e.g., Zimbra was acquired by Yahoo, Struts is developed by Apache foundation, GlassFish is developed mainly by SUN). Also, we found several instances when the open-source developers correctly used the new APIs, so they certainly were aware of the new APIs.

We can hypothesize that the open-source developers did not convert to the new APIs because the new APIs would have required creational methods which had side effects. Therefore, we conservatively only count those cases when the creational method is guaranteed not to have side-effects (e.g. the value to be inserted in the map is produced by simply instantiating a Java collection class). Even so, Table 3 shows that the open-source developers missed several opportunities to use the new APIs. CONCURRENCER missed much fewer opportunities. These are all rare, intricate patterns currently not supported by CONCURRENCER, but they could all be supported by putting more engineering effort in the tool.

\subsection{Q3: What is the speedup of the par- allelized algorithms?}

Table 4 shows the speedup of the parallelized algorithms $\left(\right.$ speedup $=$ time $_{\text {seq }} /$ time $\left._{\text {par }}\right)$. For the sorting algo-

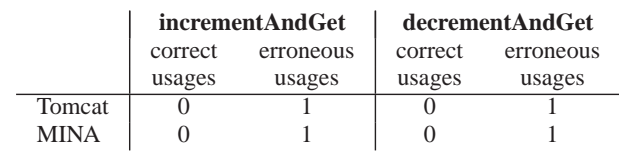

\section{Table 2. Human errors in using AtomicInteger updates in refactorings performed by open-source developers.}

\begin{tabular}{l|cc}
\multirow{2}{*}{ program } & \multicolumn{2}{|c}{ speedup } \\
& $\mathbf{2}$ cores & $\mathbf{4}$ cores \\
\hline mergeSort & $1.18 \mathrm{x}$ & $1.6 \mathrm{x}$ \\
fibonacci & $1.94 \mathrm{x}$ & $3.82 \mathrm{x}$ \\
maxSumConsecutive & $1.78 \mathrm{x}$ & $3.16 \mathrm{x}$ \\
matrixMultiply & $1.95 \mathrm{x}$ & $3.77 \mathrm{x}$ \\
quickSort & $1.84 \mathrm{x}$ & $3.12 \mathrm{x}$ \\
maxTreeDepth & $1.55 \mathrm{x}$ & $2.38 \mathrm{x}$ \\
\hline Average & $1.7 \mathrm{x}$ & $2.97 \mathrm{x}$
\end{tabular}

\section{Table 4. Speedup of the parallelized divide- and-conquer algorithms.}

rithms we use random arrays with 10 million elements. For fibonacci we compute the fibonacci value for the number 45 . For maxsumconsecutive we use an array with 100 million random integers. For matrixMultiply we use matrices with $1024 \times 1024$ doubles. For maxTreeDepth we use a dense tree of depth 50 .

\section{Related Work}

The earliest work on interactive tools for parallelization stemmed from the Fortran community and it targets loop parallelization. Interactive tools like PFC [9], ParaScope [11], and SUIF Explorer [14] rely on the user to specify what loops to interchange, align, replicate, or expand, what scalars to vectorize, etc. ParaScope and SUIF Explorer visually display the data dependences. The user must either determine that each loop dependence shown is not valid (due to conservative analysis in the tool), or transform a loop to eliminate valid dependences.

Freisleben and Kielman [5] present a system that parallelizes divide-and-conquer $\mathrm{C}$ programs, similar in spirit to our Convert Recursion to FJTAsk refactoring. To use their system, a programmer annotates (i) what computations are to be executed in parallel, (ii) the synchronization points after which the results of the subproblems are expected to be available, (iii) the input and output parameters of the recursive function, and (iv) the sequential threshold. The annotated program is preprocessed and transformed into a program which uses message-passing to communicate between the slave processes that execute the subproblems. Unlike their system, CONCURRENCER is not restricted to algorithms that 


\begin{tabular}{|c|c|c|c|c|c|c|}
\hline & \multicolumn{3}{|c|}{ putIfAbsent } & \multicolumn{3}{|c|}{ remove } \\
\hline & $\begin{array}{l}\text { potential } \\
\text { usages }\end{array}$ & $\begin{array}{c}\text { human } \\
\text { omissions }\end{array}$ & $\begin{array}{c}\text { CONCURRENCER } \\
\text { omissions }\end{array}$ & $\begin{array}{l}\text { potential } \\
\text { usages }\end{array}$ & $\begin{array}{c}\text { human } \\
\text { omissions }\end{array}$ & $\begin{array}{c}\text { CONCURRENCER } \\
\text { omissions }\end{array}$ \\
\hline MINA & 0 & 0 & 0 & 0 & 0 & 0 \\
\hline Tomcat & 0 & 0 & 0 & 0 & 0 & 0 \\
\hline Struts & 6 & 1 & 0 & 0 & 0 & 0 \\
\hline GlassFish & 7 & 3 & 1 & 6 & 5 & 0 \\
\hline JaxLib & 11 & 2 & 0 & 0 & 0 & 0 \\
\hline Zimbra & 49 & 27 & 9 & 4 & 3 & 0 \\
\hline Total & 73 & 33 & 10 & 10 & 8 & 0 \\
\hline
\end{tabular}

Table 3. Human and Concurrencer omissions in using ConcurrentHashMap's put IfAbsent and conditional remove.

use only two recursive subdivisions of the problem, and CONCURRENCER automatically infers all the parameters of the transformation (except the sequential threshold).

Bik et al. [2] present Javar, a compiler-based, sourceto-source restructuring system that uses programmer annotations to indicate parallelization of loops and of recursive algorithms. Javar rewrites the annotated code to run in parallel using multiple threads. Javar's support for parallelizing recursive functions is not optimal: each recursive call forks a new thread, whose overhead can be greater than the useful computation. Unlike Javar, (i) CONCURRENCER does not require any programmer annotations, (ii) the parallel recursion benefits from the efficient scheduling and load-balancing of the FJTask framework, and (iii) we report on experiences with using CONCURRENCER to parallelize several divide-and-conquer algorithms.

Vaziri et al. [16] present a data-centric approach to making a Java class thread-safe. The programmer writes annotations denoting atomic sets, i.e., sets of class fields that should be updated atomically, and units-of-work, i.e., methods operating on atomic sets that should execute without interleaving from other threads. Their system automatically generates one lock for each atomic set and uses the lock to protect field accesses in the corresponding units-of-work. Their system eliminates data races involving multiple variables, whereas CONCURREncer works with AtomicInteger and Concurrent HashMap that are designed to protect only single-variables. However, Concurrencer does not require any programmer annotations.

Balaban et al. [1] present a tool for converting between obsolete classes and their modern replacements. The programmer specifies a mapping between the old APIs and the new APIs, and the tool uses a type-constraint analysis to determine whether it can replace all usages of the obsolete class. Their tool is more general than ours, since it can work with any API mapping, for example one between HashMap and Concurrent HashMap. ConcurRencer is less general, since the conversion between HashMap and ConcurrentHashMap is custom implemented. However, such a conversion requires more powerful AST pattern matching and rewriting than the one used in their tool. Their tool can replace only a single API call at a time, whereas our tool replaces a set of related but dispersed API calls (like the ones in Fig. 2, 3).

Boshernitsan et al. [3] present $\mathrm{iXj}$, a general framework for code transformations. $\mathrm{iXj}$ has an intuitive user interface that enables the user to quickly sketch a pattern for the code transformation. Although useful for a broad range of transformations, $\mathrm{iXj}$ is not able to transform code where the pattern matching executes against several dispersed statements (like the ones in Fig. 2, 3, 5). In such scenarios, a user needs to use a custom implemented transformation tool like CONCURRENCER.

\section{Conclusions and Future Work}

Refactoring sequential code to concurrency is not trivial. A good way to introduce concurrency into a program is via use of a good concurrency library such as j.u.c.. Reengineering existing programs in this way is still tedious and error-prone.

Even seemingly simple refactorings_-like replacing data types with thread-safe, scalable implementations-is prone to human errors. In this paper we present CONCURRENCER, which automates three refactorings for converting integer fields to AtomicInteger, for converting hash maps to ConcurrentHashMap, and for parallelizing divide-andconquer algorithms. Our experience with ConCurrencer shows that it is more effective than a human developer in identifying and applying such transformations, and the parallelized code exhibits good speedup.

We plan to extend Concurrencer to support many other features provided by j.u.c.. Among others, ConcurRENCER will convert sequential code to use other threadsafe Atomic* and scalable collection classes, will extract other kinds of computations to parallel tasks using the Executors framework (task parallelism), and will convert Arrays to ParallelArrays, a construct which enables parallel execution of loop operations (data parallelism).

As library developers make better concurrent libraries, the "introduce concurrency" problem will become the "in- 
troduce a library" problem. Tool support for introducing such concurrent libraries is crucial for the widespread use of such libraries, resulting in more thread-safe, more scalable programs.

\section{References}

[1] I. Balaban, F. Tip, and R. Fuhrer. Refactoring support for class library migration. In OOPSLA '05: Proceedings of Object-oriented programming, systems, languages, and applications, pages 265-279, New York, NY, USA, 2005. ACM Press.

[2] A. J. C. Bik, J. E. Villacis, and D. Gannon. javar: A prototype java restructuring compiler. Concurrency - Practice and Experience, 9(11):1181-1191, 1997.

[3] M. Boshernitsan, S. L. Graham, and M. A. Hearst. Aligning development tools with the way programmers think about code changes. In CHI '07: Proceedings of the SIGCHI Conference on Human Factors in Computing Systems, pages 567-576, New York, NY, USA, 2007. ACM.

[4] D. Dig, J. Marrero, and M. D. Ernst. How do programs become more concurrent? A story of program transformations. Technical Report MIT-CSAIL-TR-2008-053, MIT, September 2008.

[5] B. Freisleben and T. Kielmann. Automated transformation of sequential divide-and- conquer algorithms into parallel programs. Computers and Artificial Intelligence, 14:579-596, 1995.

[6] M. Frigo, C. E. Leiserson, and K. H. Randall. The implementation of the cilk-5 multithreaded language. In PLDI '98: Proceedings of the ACM SIGPLAN 1998 conference on Programming language design and implementation, pages 212223, New York, NY, USA, 1998. ACM.

[7] B. Goetz. What's New for Concurrency on the Java Platform. Keynote Talk at JavaOne Conference, 2008. http: //developers.sun.com/learning/javaoneonline/j1sessn. jsp?sessn $=$ TS $-5515 \& \mathrm{yr}=2008 \&$ track $=$ javase.

[8] B. Goetz, T. Peierls, J. Bloch, J. Bowbeer, D. Holmes, and D. Lea. Java Concurrency in Practice. Addison-Wesley, 2006.

[9] J.R.Allen and K. Kennedy. PFC: A program to convert Fortran to parallel form. In Supercomputers: Design and Applications, pages 186-205, 1984.

[10] JSR-166y Specification Request for Java 7. http://g.oswego.edu/dl/concurrency-interest/.

[11] K. Kennedy, K. S. McKinley, and C.-W. Tseng. Analysis and transformation in the parascope editor. In ICS, pages 433-447, 1991.

[12] D. Lea. Concurrent Programming in Java. Second Edition: Design Principles and Patterns. Addison-Wesley, 1999.

[13] D. Lea. A java fork/join framework. In JAVA '00: Proceedings of the ACM 2000 conference on Java Grande, pages 3643, New York, NY, USA, 2000. ACM.

[14] S.-W. Liao, A. Diwan, J. Robert P. Bosch, A. Ghuloum, and M. S. Lam. Suif explorer: an interactive and interprocedural parallelizer. SIGPLAN Not., 34(8):37-48, 1999.

[15] R. Rugina and M. C. Rinard. Automatic parallelization of divide and conquer algorithms. In PPOPP, pages 72-83, 1999.
[16] M. Vaziri, F. Tip, and J. Dolby. Associating synchronization constraints with data in an object-oriented language. In $P O P L$ '06: Conference record of the 33rd ACM SIGPLAN-SIGACT symposium on Principles of programming languages, pages 334-345, New York, NY, USA, 2006. ACM. 